\title{
Probing the nonlinear structure of general relativity with black hole binaries
}

\author{
K. G. Arun, ${ }^{1}$ B. R. Iyer, ${ }^{1}$ M. S. S. Qusailah, ${ }^{1, *}$ and B. S. Sathyaprakash ${ }^{2}$ \\ ${ }^{1}$ Raman Research Institute, Sadashivanagar, Bangalore 560 080, India \\ ${ }^{2}$ School of Physics and Astronomy, Cardiff University, Cardiff, CF24 3YB, United Kingdom
}

(Received 10 March 2006; published 6 July 2006)

\begin{abstract}
Observations of the inspiral of massive binary black holes $(\mathrm{BBH})$ in the Laser Interferometer Space Antenna (LISA) and stellar mass binary black holes in the European Gravitational Wave Observatory (EGO) offer an unique opportunity to test the nonlinear structure of general relativity. For a binary composed of two nonspinning black holes, the nonlinear general relativistic effects depend only on the masses of the constituents. In a recent paper, we explored the possibility of a test to determine all the postNewtonian coefficients in the gravitational wave phasing. However, mutual covariances dilute the effectiveness of such a test. In this paper, we propose a more powerful test in which the various postNewtonian coefficients in the gravitational wave phasing are systematically measured by treating three of them as independent parameters and demanding their mutual consistency. LISA (EGO) will observe BBH inspirals with a signal-to-noise ratio of more than 1000 (100) and thereby test the self-consistency of each of the nine post-Newtonian coefficients that have so-far been computed, by measuring the lower order coefficients to a relative accuracy of $\sim 10^{-5}$ (respectively, $\sim 10^{-4}$ ) and the higher order coefficients to a relative accuracy in the range $10^{-4}-0.1$ (respectively, $10^{-3}-1$ ).
\end{abstract}

Binary pulsar observations provide one of the most stringent methods to test the strong field regime of gravity in general relativity (GR) and its alternatives [1]. The test is possible since the orbital dynamics of the binary is relativistic enough to allow the measurement of effects due to gravitational radiation damping at the post-Newtonian order $(v / c)^{5}$. Binary pulsar measurements are performed by fitting the pulse arrival times to a relativistic "timing" model [1,2], which is a function of the Keplerian parameters (orbital period, eccentricity and the projected semimajor axis of the pulsar orbit) and post-Keplerian (PK) parameters (the periastron advance, time-dilation, and secular change of the orbital period). Two more PK parameters, related to the Shapiro-delay caused by the gravitational field of the companion, can be measured if the orbit is seen nearly edge-on. Different theories of gravity have different predictions for the values of the PK parameters as functions of the individual masses of the binary constituents $m_{1}$ and $m_{2}$. Thus, a measurement of three or more PK parameters facilitates a test by requiring consistency, within the observational errors, in the estimation of the masses of the two bodies as determined by the various parameters. The most rigorous test possible so far is with the most relativistic binary pulsar PSR J0737-3039 [3]. Observed almost edge-on, it permitted the measurement of five PK parameters, which together with an additional constraint from the measurement of mass-ratio, determine and check the consistency of the masses of the two pulsars in the $m_{1}-m_{2}$ plane [3].

Although radio binary pulsars are capable of testing certain lower post-Newtonian (PN) order general relativistic effects, such as the advance of the periastron and the

*On leave of absence from Sana'a University, Yemen quadrupole approximation to the generation of gravitational waves, they will, unfortunately, not be able to probe the strong field nonlinear effects, such as the tails of gravitational waves [4]. This is because the PN expansion parameter is of order $v \sim 10^{-3}$-far too small for the effects that first appear at higher post-Newtonian orders to play a significant role in radio observations of binary neutron stars. Space- and ground-based gravitational wave detectors, such as the Laser Interferometer Space Antenna (LISA), Laser Interferometer Gravitational Wave Observatory (LIGO), VIRGO and European Gravitational Wave Observatory (EGO), will observe compact binary neutron stars and binary black holes (BBH) in the last stages of their nonlinear evolution, during which the parameter $v$ is 2 orders of magnitude larger $(v \sim 0.2-0.4)$ than it is for current radio observations of such systems. For some of the rare (about once per year) inspiral events observed by LISA (EGO) the amplitude signal-to-noise ratio could be as large as 3000 (100). Such high SNR events will allow us to measure the parameters of the signal and the source quite accurately, thereby allowing tests that were not feasible earlier. Different tests of GR have been proposed by various authors using $\mathrm{GW}$ observations of the inspiralling compact binaries [5-7] and contrasted with the binary pulsar observations [8]. These tests would necessitate an accurate parameter extraction [9] scheme using the highest PN order waveform available.

The GW "phasing formula" is very close in spirit to the "timing formula" used in the binary pulsar observations. The timing formula, $\phi_{n}^{\mathrm{PSR}}=F_{\mathrm{T}}\left[t_{n}, p_{i}\right]$, connects the rotational phase $\phi_{n}$ of a spinning pulsar to the time-of-arrival $t_{n}$ of the radio signal and a set of Keplerian and PK parameters $p_{i}=\left\{p^{\mathrm{K}}, p^{\mathrm{PK}}\right\}$. Similarly, a precise model for GWs from a compact binary will need accurate informa- 
tion about the continuous evolution of the GW phase. Schematically, the phasing formula reads $\phi^{\mathrm{GW}}=$ $F_{\mathrm{P}}\left[t, q_{i}\right]$ where, in Einstein's theory, $q_{i}$ carry the information of the source via functions of the individual masses and spins. The phasing formula consists of different PN parameters $q_{i}$, similar to the PK parameters of the timing formula, and is currently available up to relative 3.5PN order i.e., $\mathcal{O}\left(\boldsymbol{v}^{7}\right)[10,11]$. In the present paper we propose and explore an interesting possibility of testing general relativity with the high-SNR GW observations of $\mathrm{BBH}$ inspirals by LISA and EGO. The proposed test is similar in essence to the binary pulsar test, but in a stronger and dynamic regime of gravity. Using the two lowest order PN coefficients $q_{i}$ as basic variables to parametrize the waveform and choosing the other PN coefficients as "test" parameters, one at a time, it is possible to perform many consistency checks of the PN coefficients in the $m_{1}-m_{2}$ plane. In the rest of the paper we investigate this possibility in greater detail.

Binary black holes in close orbit around each other are highly relativistic and mandate the inclusion of higher PN order terms in their description. Gravitational waves emitted during the inspiral phase comprise a variety of terms arising from the nonlinear multipole interactions as the radiation propagates from the source to the far-zone [12]. These nonlinear interactions lead to the phenomenon of tails at orders $1.5 \mathrm{PN}$ and $2.5 \mathrm{PN}$ (propagation not only on but inside the light cone as well) and tails-of-tails at 3PN. For spinning binaries, there also exist effects of spin-orbit and spin-spin couplings at $1.5 \mathrm{PN}$ and $2 \mathrm{PN}$, respectively. These effects are imprinted in the emitted gravitational radiation and can be extracted by matching the detector data with an expected gravitational waveform, often called an optimal filter or a template. The template itself can only be computed using post-Newtonian theory in which the various physical quantities relevant to the emission of gravitational waves are expanded in an asymptotic series in the small parameter $v$ - the characteristic velocity in the system [13]. An important feature of the PN expansion is the presence of $\log$-terms $v^{m}(\ln v)^{n}$, where $m$ and $n$ are integers. General relativity is incompatible with a simple Taylor expansion in only powers of $v$. For instance, currently, the expansions of the specific binding energy $E$ and gravitational wave flux $\mathcal{F}$ are known to order $v^{7}$ (i.e. 3.5PN order) and given by

$$
\begin{gathered}
E=-\frac{1}{2} \eta v^{2} \sum_{k=0}^{3} E_{k} v^{2 k}, \\
\mathcal{F}=\frac{32}{5} \eta^{2} v^{10} \sum_{k=0}^{7} \mathcal{F}_{k} v^{k}-\frac{1712}{105} \ln (v) v^{6},
\end{gathered}
$$

where $\eta=m_{1} m_{2} / M^{2}$ is the symmetric mass ratio in terms of the total mass $M=m_{1}+m_{2}$ and where the coefficients
$E_{k}$ and $\mathcal{F}_{k}$ can be found in Ref. [14]. Note the presence of the $\log$-term at order $v^{6}$ in the expression for the flux.

To understand how we might test the nonlinear structure of general relativity let us begin with the Fourier domain representation $H(f)$ of the signal from a binary at a luminosity distance $D_{L}[15]$ consisting of black holes of masses $m_{1}$ and $m_{2}$ :

$$
H(f)=\frac{C \mathcal{M}^{5 / 6}}{D_{L} \pi^{2 / 3}} \sqrt{\frac{5}{24}} f^{-7 / 6} e^{i \Psi(f)+i \pi / 4},
$$

where $\mathcal{M}=\eta^{3 / 5} M$ is the chirp mass and $0 \leq C \leq 1$ is a constant that depends on the relative orientation of the detector and source with a root-mean-square value of $2 / 5$ when averaged over all sky locations and source orientations. The phase $\Psi(f)$ is given by

$$
\Psi(f)=2 \pi f t_{c}-\Phi_{c}+\sum_{k=0}^{7}\left[\psi_{k}+\psi_{k l} \ln f\right] f^{(k-5) / 3} .
$$

Here $t_{c}$ and $\Phi_{c}$ are the epoch of merger and the signal's phase at that epoch, respectively. The nonzero coefficients in the PN expansion of the Fourier phase can simply be read off from $\alpha_{n}$ s in Eq. (3.4) of Ref. [9]. The nonvanishing coefficients of the log-terms up to $3.5 \mathrm{PN}$ are $\psi_{5 l}=$ $-\frac{65 \pi}{384}+\frac{38645 \pi}{32256 \eta}$ and $\psi_{6 l}=-\frac{107}{42 \eta}(\pi M)^{1 / 3}$. For completeness, $\psi_{6}=\frac{3 \alpha_{6}}{128 \eta}(\pi M)^{1 / 3}-\psi_{6 l} \log f$. We have a total of nine post-Newtonian parameters, seven of these are the coefficients of $v^{n}$ terms for $n=0,2,3,4,5,6,7$ and two are coefficients of $v^{n} \ln (v)$ terms [16] for $n=5,6$ but each of these parameters depends only on the masses of the two black holes for the nonspinning case in GR.

Before proceeding with the description of our work, let us summarize the assumptions implicit in the analysis, the justification for doing so and their possible implications. As in most works on this subject, to demonstrate the "principle" of the proposed method we neglect the effects of spins and eccentricity. What will change on including these additional parameters is the accuracy of the test. The spin effects are relevant only when one of the black holes is much smaller than the other and/or when the black holes have their dimensionless spin angular momentum close to unity. It is not clear that astrophysical black holes, especially the supermassive ones, will be extreme Kerr. Except in cases where both BHs are extreme Kerr (or close to it) spin effects are less important for the proposed tests since we have considered black holes of comparable masses in our study. The issue of eccentricity, especially for certain LISA sources, is a complex issue depending on the astrophysical scenario related to formation mechanisms of the binary. Our neglect of eccentricity in these cases is a simplifying assumption at present. Finally let us comment on the use of the so-called restricted PN waveform in this work. Not merely in connection with tests that have been proposed but more seriously in most works related to the 
detection problem in GW data analysis, the late inspiral and merger part is ignored in the first instance. One begins by using state-of-the-art restricted PN inspiral templates. Restricted PN waveforms will only bring new variety (higher harmonics) without increasing the number of parameters; a full test should definitely use the full waveform. Including PN amplitude corrections could improve the tests and this is what we are doing as a follow-up of the present analysis. By the time LISA and EGO operate there could be reliable merger waveforms that can be included in the phasing and this would make this test more robust.

Given a high SNR binary black hole event one can, in principle, make a model-independent measurement of the above PN coefficients by accepting those values that best fit the data as our estimates. A procedure in which all the parameters $\boldsymbol{\theta} \equiv\left(t_{c}, \Phi_{c}, \psi_{k}, \psi_{k l}\right), k=0,2, \ldots, 7$, are independently varied to obtain the best possible fit of the signal to the data subjects general relativity to the most stringent test possible. In a recent paper, we explored the power of such a test to determine all the known coefficients to a relative accuracy of $100 \%$ or better [17]. However, this is by no means the most powerful test. This is because the covariances between the various parameters enhance the errors in their estimation, thereby diluting the effectiveness of the test.

In the present paper we have studied the accuracy with which we can measure the PN coefficients by treating at a time only three of the nine $\psi_{k}$ coefficients to be independent and taking the rest as functions of two of the three parameters. Thus, once a high SNR event is identified, we suggest to fit the data to a template wherein three terms in the PN expansion, rather than just two as in detection problem, (or all the PN terms as proposed in Ref. [17]), are treated as independent parameters. More precisely, in Einstein's GR, the tests consist in treating the parameters $\psi_{0}$ and $\psi_{2}$ as the fundamental ones from which we can measure the masses of the two black holes by inverting the relationships $\psi_{0}=\psi_{0}\left(m_{1}, m_{2}\right)$ and $\psi_{2}=\psi_{2}\left(m_{1}, m_{2}\right)$, and asking if the measurement of a third parameter, say $\psi_{6 l}=$ $\psi_{6 l}\left(m_{1}, m_{2}\right)$, is consistent with the other two. Instead of the pair $\left(\psi_{0}, \psi_{2}\right)$ one can, in principle, equally well take any other pair to be the fundamental set. The parameters $\psi_{0}$ and $\psi_{2}$, being lower order coefficients, are best determined as compared to the others and constitute our favored pair [18].

We shall consider the estimation of parameters in the ground-based EGO and space-based LISA, using covariance matrix, for which we assume the noise PSDs as given in Refs. $[19,20]$, respectively. We shall take the fundamental parameters to be $\psi_{0}$ and $\psi_{2}$ in addition to the usual extrinsic parameters $t_{c}$ and $\Phi_{c}$. We shall take the test parameter $\psi_{T}$ to be in turn $\psi_{3}, \ldots, \psi_{7}, \psi_{5 l}$ and $\psi_{6 l}$. It should be noted that there is no test corresponding to the term involving $\psi_{5}$ since it has no frequency dependence and simply redefines of the coalescence phase $\Phi_{c}$. For groundbased detectors, Advanced LIGO and EGO, the parameters include $t_{c}, \Phi_{c}$ and the three $\psi$ 's. For LISA, on the other hand, the results correspond to the case of a single detector but with amplitude modulation caused by the motion of the detector relative to the source. In this case our Fourier domain waveform will have amplitude, phase and frequency modulations due to the orbital motion of LISA and we use the waveform given in Ref. [21]. Thus for LISA, in addition to the three $\psi$ parameters related to our tests we also have the luminosity distance and the four angles related to the source's location and orientation.

The power of the tests depends on the SNR achieved for the source. In Fig. 1 we have plotted the SNR in LISA, EGO, and Advanced LIGO [9], for BBH binaries at a distance of $z=1$ for LISA and a distance of $D_{L}=$ $200 \mathrm{Mpc}$ for EGO and Advanced LIGO. In the case of EGO, we consider stellar mass BBH of equal masses with the total mass in the range $1 M_{\odot}$ to $400 M_{\odot}$, while in the case of LISA the mass range is from $10^{4} M_{\odot}$ to $10^{7} M_{\odot}$, but scaled down by $10^{4}$ so as to fit all the curves in the same plot. While the SNR in EGO can reach several 100's for sources that it might observe every once in a year, in the case of LISA the SNR could be several 1000's for the supermassive BBH sources that it is expected to observe about once per year. The SNR's in both LISA and EGO are large enough for the tests to be very powerful probes of the PN coefficients and the nonlinear effects of GR.

The lowest order parameters $\psi_{0}$ and $\psi_{2}$ are measured with the smallest errors. In the case of LISA the errors for a source at $z=1$ are of order $10^{-5}-10^{-4}$ and in the case of EGO the errors for a source at $200 \mathrm{Mpc}$ are of order $10^{-4}-10^{-3}$. Figure 2 plots the relative errors $\Delta \psi_{T} / \psi_{T}$ for various parameters $\psi_{T}$ as a function of the total mass $M$. From the plots, it is clear that the proposed tests can be

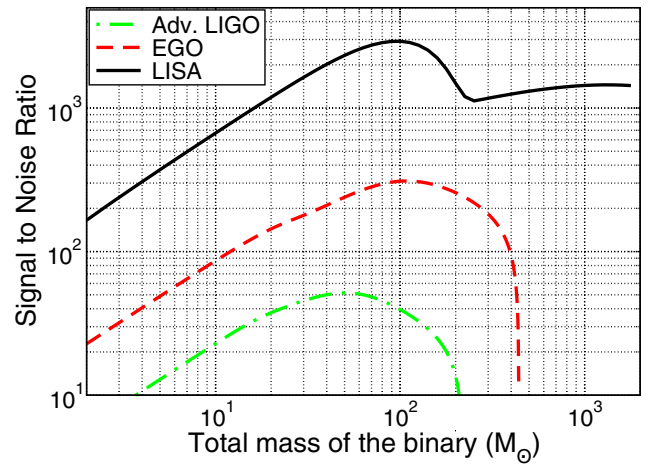

FIG. 1 (color online). The signal-to-noise ratio for stellar mass binary black holes $(\mathrm{BBH})$ in Advanced LIGO and EGO and supermassive $\mathrm{BBH}$ in LISA for equal mass binaries at a distance of $200 \mathrm{Mpc}$ (for EGO and Advanced LIGO) and $z=1$ (LISA). In the case of LISA we assume that the signal is integrated for a year (last year before coalescence) and in the case of EGO we assume that the signal is integrated over a bandwidth from $10 \mathrm{~Hz}$ until the binary reaches its innermost circular orbit. The masses of supermassive $\mathrm{BBH}$ in the case of LISA have been scaled down by a factor of $10^{4}$. 

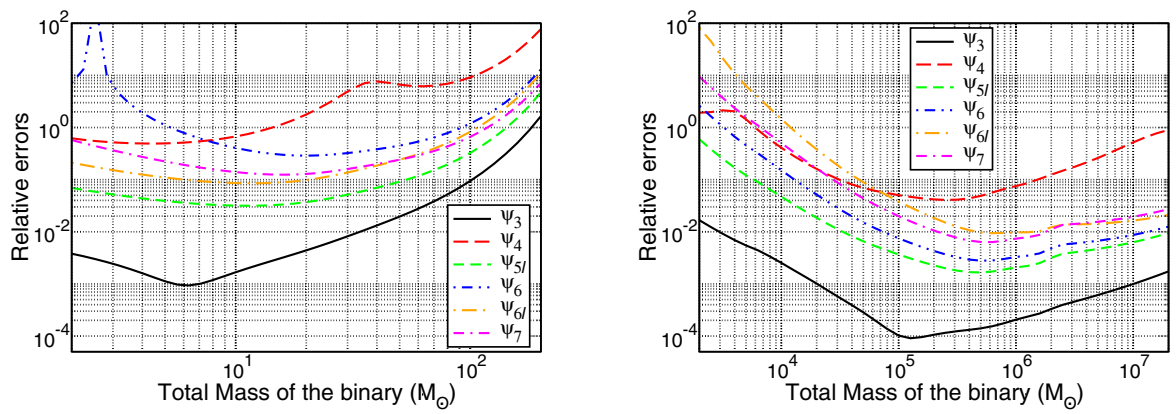

FIG. 2 (color online). Plot showing the relative errors $\Delta \psi_{T} / \psi_{T}$, in the test parameters $\psi_{T}=\psi_{3}, \psi_{4}, \psi_{5 l}, \psi_{6}, \psi_{6 l}, \psi_{7}$ as a function of the total mass $M$ of a supermassive BBH at a redshift of $z=1$ observed by LISA (right panel) and of a stellar mass compact binary at a distance of $D_{L}=200 \mathrm{Mpc}$ observed by EGO (left panel). The rest of the details as in Fig. 1.

performed effectively with all $\psi_{k}$ 's, especially in the case of LISA. This is another reason why LISA is such an important mission. All the test parameters, including the log-terms at 2.5PN and 3PN order, can be estimated with fractional accuracies better than $10^{-2}$ in the case of LISA for massive $\mathrm{BBH}$ binaries with the total mass in the range $10^{4}-10^{7} M_{\odot}$, and with fractional accuracies better than $100 \%$ in the case of EGO for stellar mass BBH binaries with the total mass range $2-10 M_{\odot}$. This demonstrates the exciting possibility of testing the nonlinear structure of general relativity using the GW observations by EGO and LISA. A similar analysis in the case of Advanced LIGO for sources with the total mass $\sim 10 M_{\odot}$, shows that all the parameters, except $\psi_{4}$ and $\psi_{6 l}$, can be measured to a relative accuracy of $100 \%$. Thus, though the 3PN logterm cannot be probed with Advanced LIGO, the 2.5PN log-term can be tested leading to an interesting possibility in the more immediate future.

With reference to Fig. 2, one may wonder why the error in $\psi_{4}$ is the largest relative to the other, higher order, $\psi$ 's. We believe that there are several reasons for this odd behavior: recall that the PN terms in the Fourier phase are given by $\psi_{k} f^{(k-5) / 3}$. When $k=5$, there is no dependence on frequency and when $k=4$ the term varies very slowly as $f^{-1 / 3}$. Therefore, terms close to $k=5$ are likely to suffer from large variances since the frequency dependence of the corresponding term is weak. Although one might expect $\psi_{6}$ also to suffer from large relative errors, the fact that in this case the term increases with frequency as $f^{1 / 3}$ contributes to making it a more important term than $\psi_{4}$. We also observe that $\psi_{4}$ has significantly larger covariances with $\psi_{0}$ and $\psi_{2}$ which adds to its poor determination.

In Fig. 3, we have depicted the power of the proposed test in the $m_{1}-m_{2}$ plane. We present the uncertainty contours, with 1- $\sigma$ error bars, associated with the different test parameters in the $m_{1}-m_{2}$ plane, when $\psi_{0}$ and $\psi_{2}$ are used to parametrize the waveform and in the case of LISA. The parameter $\psi_{6 l}$ is much better determined by LISA than EGO, as one would expect. This figure is an explicit demonstration of the efficacy of the proposed test and the accuracy with which the future $\mathrm{GW}$ observations of $\mathrm{BH}$ binaries by EGO and LISA can test GR in its strong field regime.

As mentioned earlier, the spin and angular parameters add a lot of structure to the waveform which contain additional information that can be extracted and more tests conducted. Covariance between the old and new parameters is likely to increase the error boxes but the tests become more demanding as a result of seeking consistency amongst a greater number of parameters. Future studies should look into the more general case incorporating the effects of spin and systematic effects of orbital eccentricity that could affect the tests, and more interestingly, go beyond the restricted waveform approximation by incorporating the amplitude corrections [22] to the GW phasing.

We conclude by discussing the extent to which we can extend the current proposal to discriminate between different theories of gravity such as massive graviton theories and scalar-tensor theories $[6,23]$. The limitations of GW

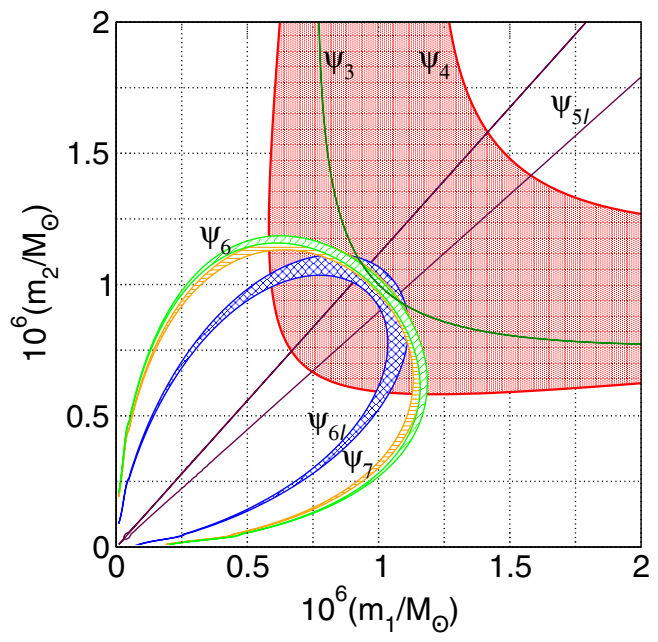

FIG. 3 (color online). Plot showing the regions in the $m_{1}-m_{2}$ plane that correspond to 1- $\sigma$ uncertainties in the test parameters $\psi_{T}=\psi_{3}, \psi_{4}, \psi_{5 l}, \psi_{6}, \psi_{6 l}, \psi_{7}$ for a $\left(10^{6}, 10^{6}\right) M_{\odot}$ supermassive black hole binary at a redshift of $z=1$ as observed for a year by LISA. (Note that the $1-\sigma$ uncertainty in $\psi_{3}$ is smaller than the thickness of the line.) 
phasing to quantitatively discriminate between alternate theories of gravity has been critically discussed in [8] and should be kept in mind. For the massive graviton theories the 1PN phasing term $\psi_{2}$ is different and also involves the Compton wavelength of the graviton $\lambda_{g}$. Using $\psi_{0}$ and $\psi_{3}$ as basic variables and $\psi_{2}$ as a test [24], we find that bounds can be set on the value of $\lambda_{g}$, modulo the neglect of uncomputed higher PN order corrections in the theory. Using EGO, which will observe stellar mass BH coalescences, we can set a bound on $\lambda_{g}$ to be $1.3 \times$ $10^{13} \mathrm{~km}$ whereas with LISA the bounds are as high as $7.12 \times 10^{16} \mathrm{~km}$. Scalar-tensor theories like Brans-Dicke theory, which predicts dipolar GW emission, have leading additional terms in the phasing formula at a PN order lower than in GR. But the dipole GW emission is more important for asymmetric binaries than it is for equal mass systems. However, for such systems spin effects are also expected to play a crucial role. The present paper deals with only nonspinning binaries and we postpone the questions relating to dipolar radiation including spin effects to a future work. Once again, these tests will be limited by the uncomputed higher order PN contributions in the BransDicke theory.

B. R. I. thanks the University of Wales and Cardiff, U.K. for supporting his visit in January 2006 and B. S. S. thanks the Raman Research Institute, India for hospitality during August 2005 when part of the work was carried out.
[1] T. Damour and J. H. Taylor, Phys. Rev. D 45, 1840 (1992).

[2] T. Damour and N. Deruelle, Ann. Inst. Henri Poincaré, A 43, 107 (1985); 44, 263 (1986); J.H. Taylor and J. M. Weisberg, Astrophys. J. 253, 908 (1982).

[3] A. G. Lyne et al., Science 303, 1153 (2004).

[4] L. Blanchet and G. Schäfer, Classical Quantum Gravity 10, 2699 (1993).

[5] L. Blanchet and B.S. Sathyaprakash, Classical Quantum Gravity 11, 2807 (1994); Phys. Rev. Lett. 74, 1067 (1995).

[6] C. M. Will, Phys. Rev. D 50, 6058 (1994); Phys. Rev. D 57, 2061 (1998).

[7] E. Berti, A. Buonanno, and C. M. Will, Phys. Rev. D 71, 084025 (2005).

[8] T. Damour and G. Esposito-Farèse, Phys. Rev. D 58, 042001 (1998).

[9] K. G. Arun, B. R. Iyer, B. S. Sathyaprakash, and P. A. Sundararajan, Phys. Rev. D 71, 084008 (2005).

[10] L. Blanchet, T. Damour, B. R. Iyer, C. M. Will, and A. G. Wiseman, Phys. Rev. Lett. 74, 3515 (1995).

[11] L. Blanchet, B. R. Iyer, and B. Joguet, Phys. Rev. D 65, 064005 (2002); L. Blanchet, G. Faye, B. R. Iyer, and B. Joguet, Phys. Rev. D 65, 061501(R) (2002); L. Blanchet, T. Damour, G. Esposito-Farèse, and B. R. Iyer, Phys. Rev. Lett. 93, 091101 (2004); Phys. Rev. D 71, 124004 (2005).

[12] L. Blanchet and T. Damour, Phys. Rev. D 37, 1410 (1988); L. Blanchet, Classical Quantum Gravity 15, 113 (1998); 15, 89 (1998).

[13] We use a system of units in which $c=G=1$.

[14] T. Damour, B. R. Iyer, and B. S. Sathyaprakash, Phys. Rev. D 63, 044023 (2001); 72, 029902(E) (2005).

[15] Throughout the paper we assume a cosmological model with zero spatial curvature $\left(\Omega_{\kappa}=0, \Omega_{\Lambda}+\Omega_{M}=1\right)$ and Hubble's constant to be $H_{0}=70 \mathrm{Kms}^{-1} \mathrm{Mpc}^{-1}$. The luminosity distance is given by $D_{L}=\frac{1+z}{H_{0}} \times$ $\int_{0}^{z} \frac{d z^{\prime}}{\left[\Omega_{M}\left(1+z^{\prime}\right)^{3}+\Omega_{\Lambda}\right]^{1 / 2}}$, where $z$ denotes the redshift of the
source.

[16] In the Fourier domain the log-terms appear at 2.5PN order rather than $3 \mathrm{PN}$ because of an integration that involves $1 / f$.

[17] K. G. Arun, B. R. Iyer, M.S.S. Qusailah, and B.S. Sathyaprakash, Classical Quantum Gravity 23, L37 (2006).

[18] One could alternatively use the entire PN expansion up to some $k^{\prime}$ as a basis, and then check consistency with $\psi_{k^{\prime}+1}$ (ignoring higher order terms). This would be relevant for testing alternative theories where deviations from GR grow larger with stronger gravity. However, the systematic effects from the neglect of known higher order PN corrections to GR might contaminate the difference between the alternate theory and GR in this case.

[19] M. Punturo (private communication). Fit by A. Sengupta and C. Van Den Broeck (unpublished). EGO is imagined to be a possible third generation gravitational wave detector. http://www.astro.cf.ac.uk/geo/ego/.

[20] L. Barack and C. Cutler, Phys. Rev. D 70, 122002 (2004).

[21] C. Cutler, Phys. Rev. D 57, 7089 (1998).

[22] K. G. Arun, L. Blanchet, B. R. Iyer, and M. S. S. Qusailah, Classical Quantum Gravity 21, 3771 (2004).

[23] T. Damour and G. Esposito-Farèse, Classical Quantum Gravity 9, 2093 (1992).

[24] Given the theoretical status of the phasing formula in the massive graviton case, this choice we implement is the most convenient one at present. In a more complete theory $\psi_{3}$ could in general depend on the Compton wavelength as well and there would be not much rationale behind the choice proposed. One could then use $\psi_{0}$ and $\psi_{2}$ as basic variables and $\psi_{3}$ as a test, as before. 